\title{
Radiation exposure from computerized tomography and risk of childhood leukemia: Finnish register-based case-control study of childhood leukemia (FRECCLE)
}

\author{
Atte Nikkilä, ${ }^{1}$ Jani Raitanen, ${ }^{2,3}$ Olli Lohi ${ }^{1,4}$ and Anssi Auvinen ${ }^{2,3,5}$ \\ ${ }^{1}$ Faculty of Medicine and Biosciences, University of Tampere; ${ }^{2}$ Faculty of Social Sciences, University of Tampere; ${ }^{3} U K K$ Institute for \\ Health Promotion Research, Tampere; ${ }^{4}$ Tampere Center for Child Health Research, University of Tampere and Tampere University \\ Hospital and ${ }^{5}$ STUK - Radiation and Nuclear Safety Authority, Helsinki, Finland
}

With reference to the article Nikkilä A, Raitanen J, Lohi $\bigcirc$, Auvinen A. Radiation exposure from computerized tomography and risk of childhood leukemia: Finnish register-based case-control study of childhood leukemia (FRECCLE). Haematologica. 2018;103(11):1873-1880, it has come to the authors' attention that the reported total number of computed tomography (CT) scans in our article was incorrect. The reported numbers were, in fact, the expected numbers prior to the CT scan collection from hospitals. Fortunately, this does not affect the number of CT scans to the study subjects and, thus, all results regarding childhood leukemia remain unchanged.

The errors and their corrections are listed below.

\section{An incorrect sentence appeared in the November 2018 issue, page 1876.}

Data on a total of 80,783 pediatric CT scans were obtained and of those, 49 CT scans were performed on the study subjects, excluding the 2 -year latency period (Table 1). Half $(n=25)$ were head scans, and 19 were lung scans. Of the CT scans, 36 were performed on 15 (1.4\%) cases and 13 scans on $10(0.3 \%)$ controls.

\section{The corrected version of the sentence is published below.}

Data on a total of 72,673 pediatric CT scans were obtained and of those, 49 CT scans were performed on the study subjects, excluding the 2-year latency period (Table 1). Half $(n=25)$ were head scans, and 19 were lung scans. Of the CT scans, 36 were performed on 15 (1.4\%) cases and 13 scans on $10(0.3 \%)$ controls.

\section{An incorrect version of Table 1 appaered in the November 2018 issue, page 1875. The corrected version of Table 1 is published below.}

Table 1. The collection and availability of electronically stored computed tomography scans.

\begin{tabular}{lccc} 
Hospital & City & Data availability & Number of CT scans \\
Helsinki University Hospital & Helsinki & $1990-2011$ & 28,459 \\
Tampere University Hospital & Tampere & $1978-2011$ & 17,077 \\
\hline Oulu University Hospital & Oulu & $1993-2011$ & 8,722 \\
Turku University Hospital & Turku & $1996-2011$ & 5,806 \\
\hline Kuopio University Hospital & Kuopio & $1996-2011$ & 4,115 \\
Central Finland Central Hospital & Jyväskylä & $2002-2011$ & 1,635 \\
\hline Satakunta Central Hospital & Pori & $1995-2011$ & 2,032 \\
Seinäjoki Central Hospital & Seinäjoki & $1999-2011$ & 1,347 \\
\hline Päijänne Tavastia Central Hospital & Lahti & $2000-2011$ & 447 \\
North Karelia Central Hospital & Joensuu & $1993-2011$ & 3,033 \\
\hline TOTAL & & & 72,673 \\
\hline
\end{tabular}

All Finnish university hospitals are listed first and separated slightly are the five chosen central hospitals. The reported numbers represent the data obtained before exclusions or harmonization. 
An incorrect version of Figure 2 appeared in the November 2018 issue, page 1875. The corrected version of Figure 2 is published below.

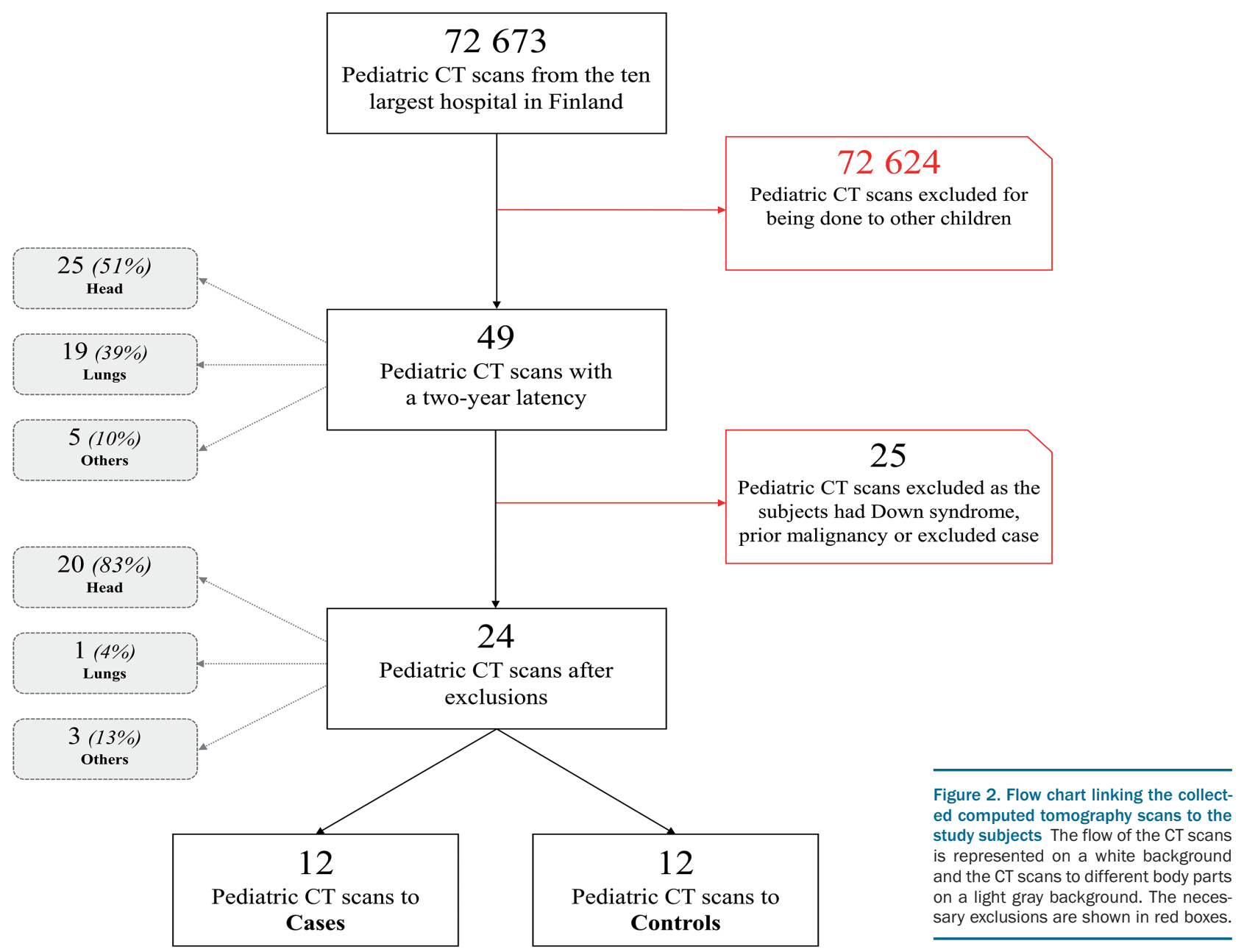

